\title{
A CLINICAL AND BIOCHEMICAL STUDY OF THE SUBNORMAL CHILD
}

\author{
BY \\ A. ARNOLI) OSMAN, M.R.C.P., \\ and \\ HAROLD G. CLOSE, M.B., B.S.
}

(From the Medical Investigation Dept., Queen Mary's Hospital, E.)

The subjects discussed in this paper are the results of an investigation primarily undertaken with a view to determining as far as possible some of the biochemical factors underlying, and predisposing to, the various forms of nephritis in children and adults. In the course of our investigations, it soon became evident that the problem resolved itself into a study of subnormal health or 'debility,' as predisposing to disease in general; or, in other words, into a a study of the nature of the soil upon which disease flourishes. In this paper it is proposed to present the results of the investigation in so far as they relate particularly to children. The results of parallel observations on adults are being published elsewhere ${ }^{1}$. It is hoped too, that some of the results obtained may form a standard upon which to base further observations on specific diseases as they occur in children, for not a few biochemical data,published as representing the variations peculiar to specific diseases, are in fact already present in that state of subnormal health. This so frequently precedes frank disease that it in part explains the similarity in the findings in diseases which are, in their pathogenesis and clinical nature, widely different.

Selection of cases.-The children investigated were all of the elementary day-school social class, who attended the medical out-patient department of one of us (A.A.O.), at Queen Mary's Hospital, for such minor symptoms as headache, lassitude, constipation, and bilious attacks. The actual symptoms for which advice was sought are given in Table 1 . The children were all between the ages of six to twelve years inclusive. Those who had recently suffered from some acute illness, such as tonsillitis or measles, were not included.

The normal control children weire selected from those patients who attended the casualty department for such conditions as ringworm, or uninfected cut fingers, and who appeared in every other way to be in perfectly normal health. A certain number of normals was also recruited from among the families of patients.

In this way it was found possible to divide the cases roughly into two groups clinically, the normals, and the subnormals or 'debilitated.' A certain amount of overlapping between the groups inevitably occurred, as it was desired particularly to investigate that borderland state between health and 
disease, where the one passes almost imperceptibly into the other, but as will be seen later, subsequent biochemical observations to a large extent confirmed and justified this method of selection.

With few exceptions, a sample of blood for examination was taken between two and three hours after the last meal, the nature and constitution of which was ascertained. Measures were also adopted to ensure that each child was resting quietly for at least an hour before the blood was taken, and of course, in no case was the child aware that this operation was to follow. After considerable experience in obtaining blood from children, it is considered that the technique employed is so free from causing pain, and even but momentary apprehension, that changes in blood chemistry from this cause can be excluded. In a few instances where the element of fright was encountered, the cass was excluded from the series.

Methods. - The following methods were used in this investigation :-

(1) Plasma bicarbonate, by the method of Van Slyke, Stillman and Cüllen². Titration was carried to a constant end-point of $\mathrm{pH}=7 \cdot 4$. Phenol red was used as indicator. Results are expressed as molar concentration of bicarbonate.

(2) Plasma chlorides by the method of Claudius ${ }^{3}$, results being expressed as grammes of $\mathrm{NaCl}$ per 100 c.cm.

(3) Ketone bodies in the blood by the method of Van Slyke and Fitz

(4) Blood sugar (venous blood), by the method of Folin and $\mathrm{Wu}$, results being expressed as milligrammes per 100 c.cm.

Procedure.-The following procedure was adopted, by means of which it is hoped that any personal bias in correlating the biochemical and clinical results, has been reduced to a minimum.

Each child was submitted to a thorough clinical examination by one of us (A.A.O.). This included inspection of the tonsils, etc., and a qualitative examination of the urine for albumen, sugar, and acetone bodies (Rothera and ferric chloride reactions). These results, with the history of the case, and notes on signs or symptoms, were entered up, and a diagnosis of either ' normal,' or 'subnormal' was made. The case was numbered and the child was given a card bearing this number only.

The collaborator (H.G.C.), who had not previously seen the child, then took, without undue compression, a sample of blood under paraffin from a vein in the arm into an oxalated centrifuge tube, which was correspondingly numbered. Estimations were performed within twelve hours of taking the blood, it having been found previously that none of the quantities estimated showed appreciable variations within this period. The blood sugar, however, was estimated immediately after taking the sample of blood.

\section{Comparison of normal and subnormal children.}

The main clinical features and chief symptoms of all the children investigated in this series are shown in Table 1. A few of the headings in this table require explanation. Cyclical vomiting ('C.V.') was diagnosed only when there was a clear history of recurrent 'bilious attacks,' preceded by listlessness, and accompanied by headache, vomiting and prostration, lasting 48 hours or more, and necessitating confinement to bed for this period. Debility (' D') was diagnosed in those children who were in subnormal health, but who did not give a clear history of attacks of cyclical vomiting. Children who were in a permanent state of debility, and who also suffered from cyclical vomiting, have been olassed under the heading of C.V. only. 
The nomenclature adopted in assessing the condition of the tonsils was as follows:-Cases in whom a complete tonsillectomy had been performed at least a year previously, were labelled (a); those in whom there was no history at any time of throat or ear trouble, and whose tonsils appeared to be normal, were labelled (b) ; and those who gave a history of repeated throat troubles, i.e., tonsillitis, 'sore throats,' cervical adenitis, with or without chronic otorrhœa, and whose tonsils either appeared unduly large, or definitely septic, were labelled (c). No doubt this classification is open to criticism, but it was the best that could be devised, and the number of cases is sufficiently large to make it at least of some value.

An attempt was also made to assess roughly the temperament of each child, that is, whether or not there was an undue tendency to nervousness, excitability, or the reverse. In doing so it was only possible to form an opinion from the behaviour of the child under examination, and from the parent's statements regarding this-neither of them very reliable guides.

Table 1 shows that 108 children were investigated, 38 normals, and 70 subnormals.

Table 2 compares the blood sugar, plasma bicarbonate, and chloride, in groups of normal and subnormal children.

From a consideration of these figures (Table 2), three important facts emerge. First, the plasma bicarbonate is considerably lower in the subnormal than in the normal children. In fact, a definite 'acidosis' is the rule in debilitated children, if the term 'acidosis' is defined as simply a decrease in the plasma bicarbonate, however produced. Such a definition does not necessarily imply any actual change in reaction, or $\mathrm{pH}$, in the direction of increased acidity, in the strict chemical sense of the word. Secondly, this acidosis of debility, is associated with a slight, but definite, increase of the plasma chloride, compared with that of the normals. This point is of interest in view of the experiments of Haldane ${ }^{5}$ and his co-workers, who showed that after ammonium chlorids ingestion, the plasma bicarbonate was decreased in exact proportion as the chloride content increased; and also because of the possibility of an accompanying increase in the water content of the body, a subject which has been more fully discussed elsewhere. ${ }^{1}$ Thirdly, it is interesting to note that the blood sugar is practically the same in both groups. This is contrary to recent teaching, which asserts that the subnormal child shows a tendency to continued hypoglycæmia-an hypothesis which seems based on insufficient evidence.

One of us (A.A.O.) when working on the subject of cyclical vomiting in children some years ago, showed that this common disorder could generally be prevented or cured by increasing the allowance of sugar in the diet, and it was assumed that there was therefore a shortage of sugar in the blood. The term ' glycopenia,' which unfortunately is still used by some, was coined to describe these children. It is clear, however, from the evidence already presented, that this term is inaccurate, and should no longer be used in this connection. As has been pointed out before ${ }^{6}$, the essential feature of these cases appears to be a shortage of the glycogen stored in the liver, and perhaps 
TABle 1. Comparisos of Normal and Subnokmal Childrex.

\begin{tabular}{|c|c|c|c|c|c|c|c|c|c|c|c|c|}
\hline \multirow[t]{2}{*}{ Case } & \multirow[t]{2}{*}{ Sex } & \multirow[t]{2}{*}{ Age } & \multicolumn{2}{|c|}{ Height } & \multicolumn{2}{|c|}{ Weight } & \multirow[t]{2}{*}{$\begin{array}{l}\text { Diag- } \\
\text { nosis }\end{array}$} & \multirow[t]{2}{*}{ Chief symptom } & \multirow[t]{2}{*}{$\begin{array}{r}\text { Consti- } \\
\text { pation }\end{array}$} & \multirow[t]{2}{*}{$\begin{array}{c}\text { Head- } \\
\text { ache }\end{array}$} & \multirow[t]{2}{*}{$\begin{array}{l}\text { Tonsil } \\
\text { history }\end{array}$} & \multirow[t]{2}{*}{ Disposition } \\
\hline & & & & in. & st. & lb. & & & & & & \\
\hline 1 & M & 10 & & & & & $\mathbf{N}$ & & & + & b & Bright \\
\hline 2 & $\mathbf{F}$ & 9 & & & & & $\mathrm{SN}$ & C.V. & 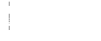 & + & & Dull \\
\hline 3 & $\mathbf{F}$ & 13 & & & & & SN & Languor & . & + & & Dull \\
\hline 4 & M & 8 & & & & & SN & C.V. & & & & Normal \\
\hline 5 & $F$ & $6 \frac{1}{2}$ & & & & & $\mathrm{SN}$ & C.V. & & + & c & Normal \\
\hline 6 & $\mathbf{F}$ & 9 & & & & & $\mathrm{~N}$ & C.V. & & & & \\
\hline 7 & $\mathbf{M}$ & 8 & & & & & SN & C.V. & & & & \\
\hline 8 & $\mathbf{F}$ & $7 \frac{1}{2}$ & & & & & SN & Headaches & & + & 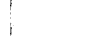 & \\
\hline 9 & $\mathbf{F}$ & 7 & & & & & SN & C.V. & & & & . \\
\hline 10 & $\mathbf{M}$ & $5 \frac{1}{2}$ & & & & & SN & C.V. & & & & Dull \\
\hline 11 & $\mathbf{F}$ & 6 & & & & & SN & C.V. & & & & \\
\hline 12 & $\mathbf{M}$ & 6 & & & & $10 \frac{1}{2}$ & SN & C.V. & & & & \\
\hline 13 & $\mathbf{F}$ & 6 & & & & $8 \frac{1}{2}$ & $\mathbf{N}$ & Languor & & - & & Dull \\
\hline 14 & $\mathbf{M}$ & 8 & & & & & SN & Languor & & + & i & Dull \\
\hline 15 & $\mathbf{M}$ & 8 & 4 & $1 \frac{1}{2}$ & 4 & $0 \frac{1}{2}$ & $\mathrm{~N}$ & & & & & Normal \\
\hline 16 & $\mathbf{M}$ & $7 \frac{1}{2}$ & & & & & $\mathrm{SN}$ & Languor & & & & Dull \\
\hline 17 & M & 12 & & & & & $\mathrm{~N}$ & C.V. & & & 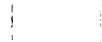 & \\
\hline 18 & M & 12 & & & & & $\mathrm{SN}$ & C.V. & & c & c & Normal \\
\hline 19 & $\mathbf{M}$ & 8 & & & & & SN & Languor & & & & Excitable \\
\hline 20 & $\mathrm{~F}$ & 12 & & & & & $\mathrm{SN}$ & C.V. & & & c & Excitable \\
\hline 22 & M & 13 & & & & & SN & C.V. & & + & 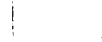 & \\
\hline 23 & $\mathbf{M}$ & 6 & & & & & SN & C.V. & & & 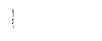 & \\
\hline 24 & $\mathrm{~F}$ & 12 & 4 & $6 \frac{3}{4}$ & 4 & $8 \frac{1}{2}$ & $\mathrm{~N}$ & C.V. & & + & $\mathrm{b}$ & \\
\hline 25 & M & 12 & & & 7 & 3 & $\mathrm{SN}$ & & - & + & $\mathrm{b}$ & Dreamy \\
\hline 26 & $\mathbf{M}$ & 12 & & & 5 & 1 & $\mathrm{~N}$ & $\begin{array}{l}\text { Recurrent abdomi. } \\
\text { nal pain. }\end{array}$ & 一 & + & $\mathrm{b}$ & Dull \\
\hline 27 & M & 10 & & & 5 & $1 \frac{1}{2}$ & $\mathbf{N}$ & $\begin{array}{l}\text { Recurrent abdomi. } \\
\text { nal pain. }\end{array}$ & & & b & \\
\hline 28 & $\mathbf{F}$ & 8 & & & & & SN & Joint \& limb pains & & + & $\mathrm{b}$ & \\
\hline 29 & M & 5 & & & 2 & $6 \frac{1}{2}$ & $S N$ & C.V. & & + & c & \\
\hline 30 & $\mathrm{~F}$ & 13 & 4 & $4 \frac{1}{2}$ & 8 & $4 \frac{1}{2}$ & SN & C.V. & & $+t$ & a & \\
\hline 31 & $\mathbf{F}$ & 7 & 3 & 11 & 3 & $3 \frac{1}{2}$ & $\mathbf{N}$ & $\begin{array}{l}\text { Threadworms and } \\
\text { C.V. }\end{array}$ & & & c & \\
\hline 32 & $\mathrm{~F}$ & 10 & & & 4 & 0 & $\mathbf{N}$ & Giddy attacks & + & + & b & Dull \\
\hline 33 & $\mathbf{F}$ & $8 \frac{1}{2}$ & & & 4 & 12 & $\mathrm{SN}$ & C.V. & & & a & Excitable \\
\hline 34 & $\mathrm{~F}$ & 6 & & & 1 & 13 & SN & Wasting and C.V. & & & b & \\
\hline 35 & $\mathrm{M}$ & 11 & 4 & $5 \frac{1}{4}$ & 4 & 8 & $\mathrm{SN}$ & $\begin{array}{l}\text { Abdominal pain } \\
\text { and C.V. }\end{array}$ & & + & $\mathrm{a}$ & \\
\hline 36 & $\mathrm{M}$ & 8 & & & & & $\mathbf{N}$ & & & & b & Normal \\
\hline 37 & $\mathbf{M}$ & $5 \frac{1}{2}$ & & & & & $\mathrm{SN}$ & C.V.; drowsiness & 一 & & c & $\begin{array}{l}\text { Dull but } \\
\text { excitable }\end{array}$ \\
\hline 38 & F & 8 & & & 3 & 1 & $\mathrm{SN}$ & C.V.\& excitability & & + & b & Excitable \\
\hline 39 & $\mathbf{F}$ & 8 & & & 3 & 6 & $\mathrm{SN}$ & Debility & & 一 & a & Excitable \\
\hline 40 & $\mathrm{M}$ & 7 & 3 & 10 & 2 & $13 \frac{1}{2}$ & $\mathrm{~N}$ & Cough & & + & c & Excitable \\
\hline
\end{tabular}




\begin{tabular}{|c|c|c|c|c|c|c|c|}
\hline Habit & Complexion & Remarks & $\begin{array}{l}\text { Plasma } \\
\mathrm{NaHCo}_{3}\end{array}$ & $\begin{array}{c}\text { Plasma } \\
\mathrm{NaCl}\end{array}$ & $\begin{array}{l}\text { Blood } \\
\text { sugar }\end{array}$ & $\begin{array}{l}\text { Rothera } \\
\text { urine }\end{array}$ & $\begin{array}{l}\text { Urinary } \\
\text { chloride }\end{array}$ \\
\hline Fat \& flabby & Pasty & Suffers from C.V. & .0306 & & & - & \\
\hline Normal & Pasty & & .0270 & & & sl. + & \\
\hline Normal & Good & & .0270 & & $\cdot 107$ & & \\
\hline Normal & & & .0280 & & .09 & - & \\
\hline \multirow[t]{4}{*}{ Normal } & Good & & .0305 & & $\cdot 108$ & - & \\
\hline & & & $\cdot 0277$ & & .079 & - & \\
\hline & & & .0278 & & .093 & 一 & \\
\hline & & & .0267 & & .085 & - & \\
\hline \multirow[t]{2}{*}{ Frail } & Pale & : & .0275 & & $\cdot 146$ & - & \\
\hline & & & $\begin{array}{l}.0223 \\
.0288\end{array}$ & & .078 & $\overline{+}$ & \\
\hline Normal & Pale & Growing pains & $\cdot 0308$ & & .089 & - & \\
\hline \multirow[t]{4}{*}{ Normal } & Pale & & $\cdot 0302$ & & $\cdot 125$ & & \\
\hline & & & $\cdot 0292$ & & $\cdot 139$ & 一 & \\
\hline & Pale & & .0263 & & $\cdot 101$ & - & \\
\hline & & $\begin{array}{l}\text { Suffers from angio-neurotic } \\
\text { œdema. }\end{array}$ & $\cdot 0302$ & & & - & \\
\hline Normal & Pale & & .0290 & & $\cdot 114$ & ++ & \\
\hline Thin & Pale & $\begin{array}{l}\text { Attended for ? chorea (not } \\
\text { confirmed). }\end{array}$ & $\cdot 0269$ & & $\cdot 130$ & - & \\
\hline Thin & Pale & Appendicectomy 2 years ago & $\cdot 0271$ & & $\cdot 116$ & - & \\
\hline & & & & & $\cdot 105$ & & \\
\hline & & & & & .075 & & \\
\hline Normal & Good & Sick in trains, etc. & .0290 & & .083 & 一 & \\
\hline Fat \& flabby & Good & Septic sores on body & .0262 & & $\cdot 103$ & 一 & \\
\hline Fat & Good & & $\cdot 0292$ & .614 & .081 & - & .985 \\
\hline Normal & Good & & .0299 & & .077 & 一 & \\
\hline Thin & Pasty & & .0287 & & .099 & - & \\
\hline \multirow[t]{7}{*}{ Thin } & Good & & $\cdot 0273$ & & .090 & + & \\
\hline & Pale & Tonsillectomy 8 years before & $\cdot 0303$ & & $\cdot 102$ & 一 & \\
\hline & & & $\cdot 0135$ & & .016 & 一 & \\
\hline & Pale & & .0290 & & .081 & - & \\
\hline & Pale & Tonsillectomy 1 year ago & $\cdot 0281$ & & .092 & - & \\
\hline & & & $\cdot 0279$ & & & - & \\
\hline & Pale & $\begin{array}{l}\text { Appendicitis simulated. Ton- } \\
\text { sillectomy } 7 \text { years ago. }\end{array}$ & .0285 & & $\cdot 109$ & - & \\
\hline Normal & Good & & .0309 & & $\cdot 102$ & - & \\
\hline Normal & Pale & & .0275 & & .093 & +++ & \\
\hline Thin & Pale & Pains in limbs in damp weather & $\cdot 0316$ & & .079 & - & \\
\hline Thin & Pale & $\begin{array}{l}\text { C.V. Tonsillectomy } 3 \text { years } \\
\text { ago. }\end{array}$ & .0302 & & & - & \\
\hline Thin & Fair & & .0280 & & & $一$ & \\
\hline
\end{tabular}


TABLE I-continued.

\begin{tabular}{|c|c|c|c|c|c|c|c|c|c|c|c|c|}
\hline Case & $\operatorname{sex}$ & Age & & ight & & eight & $\begin{array}{l}\text { Diag- } \\
\text { nosis }\end{array}$ & Chief symptom & $\begin{array}{l}\text { Consti- } \\
\text { pation }\end{array}$ & $\begin{array}{l}\text { Head- } \\
\text { ache }\end{array}$ & $\begin{array}{l}\text { Tonsil } \\
\text { history }\end{array}$ & Disposition \\
\hline & & & & in. & & - $1 \mathrm{~b}$. & & & & & & \\
\hline 41 & F & 7 & & & 2 & 9 & SN & Frequent colds & & + & $\mathrm{c}$ & Normal \\
\hline 42 & $\mathbf{F}$ & $5 \frac{1}{2}$ & & & 2 & 13 & SN & T's \& A's-C.V. & 一 & + & & $\begin{array}{l}\text { Excitable } \\
\text { butdrowsy }\end{array}$ \\
\hline 43 & M & 12 & 4 & $9 \frac{1}{2}$ & 5 & 7 & $\mathrm{~N}$ & & & + & & \\
\hline 44 & $\mathrm{~F}$ & 9 & & & 3 & $1 \frac{1}{2}$ & SN & Anorexia & & & c & Dull \\
\hline 45 & F & 9 & 4 & 4 & 3 & $10 \frac{1}{2}$ & SN & Debility & & & b & Dull \\
\hline 46 & $M$ & 8 & 3 & 10 & 3 & $2 \frac{1}{2}$ & SN & Asthma & + & & a & $\begin{array}{l}\text { Dull but } \\
\text { excitable. }\end{array}$ \\
\hline 47 & $\mathbf{M}$ & 8 & 4 & 2 & 4 & 1 & SN & Pains in chest and & + & + & $\mathbf{a}$ & Dull \\
\hline 48 & $\mathbf{F}$ & 8 & 3 & 10 & 3 & $0 \frac{1}{2}$ & $\mathrm{~N}$ & Tram-sickness & & + & c & Dull \\
\hline 49 & $\mathbf{M}$ & 13 & 4 & 4 & 4 & 10 & SN & Headaches & & & b & Dull \\
\hline 50 & $\mathbf{F}$ & 10 & 4 & 5 & 4 & 1 & SN & Abdominal pains & + & + & $\mathbf{b}$ & Excitable \\
\hline 51 & $F$ & 5 & & & 2 & 7 & SN & Frequent headaches & & + & a & Normal \\
\hline 52 & $\mathbf{F}$ & 10 & & & 4 & 3 & $\mathbf{N}$ & Frequent headaches & & & a & \\
\hline 53 & $\mathbf{F}$ & 13 & 4 & 10 & 6 & 0 & $\mathbf{N}$ & Cough & & + & c & Normal \\
\hline 54 & $\mathbf{M}$ & 8 & 4 & $1 \frac{1}{2}$ & 3 & $7 \frac{1}{2}$ & $\mathrm{~N}$ & ? heart disease & - & - & b & Normal \\
\hline 55 & $\mathbf{M}$ & $5 \frac{1}{2}$ & 3 & $7 \frac{3}{4}$ & 3 & $0 \frac{1}{2}$ & SN & $\begin{array}{l}\text { Languor, } \\
\text { anæmia }\end{array}$ & $t$ & + & c & Normal \\
\hline 56 & M & $7 \frac{1}{2}$ & 4 & $0 \notin$ & 3 & $10 \frac{1}{2}$ & SN & Boils and cough & - & + & b & Excitable \\
\hline 57 & $\mathbf{M}$ & $8 \frac{1}{2}$ & 4 & 1 & 3 & 7 & SN & Lassitude & $t$ & + & a & Excitable \\
\hline 58 & $\mathbf{M}$ & 8 & 3 & $11 \frac{1}{2}$ & 3 & $4 \frac{1}{2}$ & SN & C.V. & + & + & b & Excitable \\
\hline 59 & M & $6 \frac{3}{4}$ & & & 3 & 8 & $\mathrm{SN}$ & Lassitude & + & + & c & Dull \\
\hline 60 & $\mathbf{M}$ & $9 \frac{1}{2}$ & & & 4 & $9 \frac{1}{2}$ & $\mathbf{N}$ & & 一 & & a & Normal \\
\hline 61 & M & 8 & & & 2 & $13 \frac{1}{2}$ & SN & Asthma & - & - & b & Excitable \\
\hline 62 & $\mathbf{F}$ & 9 & & & 4 & 7 & SN & Abdominal pains & + & + & $\mathbf{a}$ & Exsitable \\
\hline 63 & M & 10 & 4 & $5 \frac{1}{2}$ & 4 & 13 & $\mathrm{~N}$ & Boils & - & + & $\mathbf{a}$ & Excitable \\
\hline 64 & $\mathbf{F}$ & 10 & 4 & 4 & 3 & $13 \frac{1}{2}$ & $\mathrm{SN}$ & Growing pains & - & + & a & Excitable \\
\hline 65 & $\mathbf{F}$ & 9 & 4 & 14 & 3 & 3 & SN & Boils & + & + & c & Excitable \\
\hline 66 & $\mathbf{M}$ & $5 \frac{1}{2}$ & 3 & 8 & 3 & 0 & $\mathrm{SN}$ & Anorexia & 一 & - & b & Normal \\
\hline 67 & $\mathbf{M}$ & 11 & 4 & 10 & 6 & 2 & SN & C.V. and boils & - & 一 & b & Normal \\
\hline 68 & $\mathbf{M}$ & 8 & 4 & 3 & 4 & 6 & SN & C.V. & + & + & b & Excitable \\
\hline 69 & $\mathbf{F}$ & 9 & & & 4 & 9 & SN & Anæmia & - & 一 & a & Phlegmatic \\
\hline 70 & $\mathbf{M}$ & 11 & 4 & $3 \frac{1}{2}$ & 4 & 7 & $\mathbf{N}$ & C.V. & - & + & b & Normal \\
\hline 71 & $\mathbf{M}$ & 13 & 5 & $3 \frac{1}{2}$ & 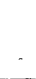 & & SN & Listlessness & + & + & a & Normal \\
\hline
\end{tabular}




\begin{tabular}{|c|c|c|c|c|c|c|c|}
\hline Habit & Complexion & Remarks & $\begin{array}{l}\text { Plasma } \\
\mathrm{NaHCo}_{3}\end{array}$ & $\begin{array}{c}\text { Plasma } \\
\mathrm{NaCl}\end{array}$ & $\begin{array}{l}\text { Blood } \\
\text { sugar }\end{array}$ & $\begin{array}{c}\text { Rothera } \\
\text { urine }\end{array}$ & $\begin{array}{l}\text { Urinary } \\
\text { chloride }\end{array}$ \\
\hline Thin & Pale & & .0260 & & & - & \\
\hline Normal & Pale & & .0269 & & & +- & \\
\hline Thin & Pale & Nervous-afraid of dark & .0321 & & & + & \\
\hline & Pale & C.V. & .0248 & & & + & \\
\hline & Pale & & .0290 & & & - & \\
\hline Thin & Pale & $\begin{array}{l}\text { Tonsillectomy } 3 \frac{1}{2} \text { years before, } \\
\text { also previous C.V. }\end{array}$ & .0280 & .594 & & 一 & \\
\hline Thin & Pale & $:$ & $\cdot 0275$ & & & - & \\
\hline Normal & Good & Nervous & .0314 & & & - & \\
\hline & Pale & $\begin{array}{l}\text { Pains in feet. Old rheumatic } \\
\text { heart. }\end{array}$ & $\cdot 0282$ & & & + & \\
\hline Thin & Good & Anorexia & $\cdot 0287$ & & & 一 & \\
\hline Normal & Good & $\begin{array}{l}\text { Pains in all joints } 2 \text { years ago. } \\
\text { Tonsillectomy } 2 \text { years ago. }\end{array}$ & .0268 & $\cdot 632$ & & - & \\
\hline Thin & Good & $\begin{array}{l}\text { Nervous. Tonsils out a year } \\
\text { ago. }\end{array}$ & .0312 & .608 & & 一 & \\
\hline Normal & Good & Sick in train & $\cdot 0314$ & 632 & & - & .655 \\
\hline Normal & Good & Ac. rheumatism 2 years ago & .0287 & .594 & & - & .702 \\
\hline Normal & Good & $\begin{array}{l}\text { Train sickness. ? occasional } \\
\text { C.V. }\end{array}$ & $\cdot 0289$ & 638 & & - & .288 \\
\hline Normal & Pale & Train sickness and C.V. & $\cdot 0277$ & $\cdot 708$ & & - & $1 \cdot 12$ \\
\hline Thin & Pale & $\begin{array}{l}\text { Train sickness. Tonsillectomy } \\
\text { l year ago. }\end{array}$ & .0275 & .623 & & - & $1 \cdot 08$ \\
\hline Thin & Good & & .0316 & 611 & & 一 & .614 \\
\hline Normal & Pale & & $\cdot 0283$ & 608 & & + & .80 \\
\hline Normal & Good & Tonsillectomy 4 years ago & .0302 & .597 & & - & .535 \\
\hline Normal & Good & & $\cdot 0277$ & 605 & & - & .807 \\
\hline Normal & Pale & $\begin{array}{l}\text { Is train sick. Tonsillectomy } 2 \\
\text { years previously, also suffers } \\
\text { from C.V. }\end{array}$ & .0294 & .620 & & 一 & .625 \\
\hline Normal & Good & $\begin{array}{l}\text { Tonsillectomy } 5 \text { years pre- } \\
\text { viously, also has cyclical } \\
\text { vomiting. }\end{array}$ & .0296 & .587 & & - & .526 \\
\hline Normal & Good & $\begin{array}{l}\text { Suffers from C.V. Tonsillec- } \\
\text { tomy } 2 \text { years before. }\end{array}$ & .0268 & .605 & & - & .658 \\
\hline Thin & Pale & Train sick & .0264 & .617 & & 一 & $\cdot 646$ \\
\hline Normal & Very pale & & .0266 & .611 & & 一 & .714 \\
\hline Normal & Good & & .0271 & .603 & & - & .453 \\
\hline Normal & Pale & $\begin{array}{l}\text { Acute rheumatism twice last } \\
\text { year. Growing pains and } \\
\text { C.V. Heart normal }\end{array}$ & .0284 & .620 & & - & $1 \cdot 14$ \\
\hline Normal & Pale & $\begin{array}{l}\text { Tonsillectomy } 1 \text { year pre- } \\
\text { viously. }\end{array}$ & -0296 & .623 & & - & $\cdot 49$ \\
\hline Normal & Good & & -0294 & 638 & & - & .439 \\
\hline Normal & Pale & $\begin{array}{l}\text { Formerly C.V. Tonsillectomy } \\
6 \text { years previously }\end{array}$ & .0283 & .594 & & 一 & .530 \\
\hline
\end{tabular}


TABLE I-continued.

\begin{tabular}{|c|c|c|c|c|c|c|c|c|c|c|c|c|}
\hline Case & Sex & Age & Hei & ight & & eight & $\begin{array}{l}\text { Diag- } \\
\text { nosis }\end{array}$ & Chief symptom & $\begin{array}{c}\text { Consti- } \\
\text { pation }\end{array}$ & $\begin{array}{l}\text { Head- } \\
\text { ache }\end{array}$ & $\mid \begin{array}{c}\text { Tonsil } \\
\text { history }\end{array}$ & Disposition \\
\hline & & & & in. & & lb. & & & & & & \\
\hline 72 & F & 8 & 3 & 9 & $3 \frac{1}{4}$ & 1 & $\mathrm{~N}$ & Headaches & - & + & $\dot{c}$ & Normal \\
\hline 73 & F & 10 & 3 & 6 & 4 & $3 \frac{1}{4}$ & $\mathrm{~N}$ & Thinness & - & - & a & Excitable \\
\hline 74 & $\mathbf{F}$ & 13 & 4 & 24 & 4 & 1 & $\mathrm{SN}$ & Pains in legs & + & - & b & Normal \\
\hline 75 & M & 9 & 4 & $2 \frac{3}{4}$ & 4 & 6 & $\mathrm{N}$ & c.v. & $t$ & + & c & Normal \\
\hline 76 & $\mathrm{~F}$ & 9 & & & 4 & 12 & $\mathrm{~N}$ & Nerves & + & - & c & $\begin{array}{l}\text { Excitable \& } \\
\text { phlegmatic }\end{array}$ \\
\hline 77 & M & $1 \%$ & 4 & 10 & 5 & 1 & SN & Headaches & - & + & b & Phlegmatic \\
\hline 78 & $\mathbf{F}$ & $7 \frac{1}{2}$ & 3 & 9 & 3 & 3 & SN & Headaches & - & + & $\mathrm{c}$ & Excitable \\
\hline 79 & M & 7 & 3 & $9 \frac{1}{2}$ & 2 & $13 \frac{1}{2}$ & SN & Catarrhs & - & + & a & Normal \\
\hline 80 & M & 7 & 4 & $1 \frac{1}{2}$ & 3 & 9 & $\mathbf{N}$ & Nerves & - & + & b & $\begin{array}{l}\text { Very ex- } \\
\text { citable }\end{array}$ \\
\hline 81 & M & 7 & 3 & 8 & $2 \frac{3}{4}$ & $13 \frac{1}{2}$ & $\mathrm{~N}$ & C.V. & - & + & c & Normal \\
\hline 82 & M & 7 & 3 & $5 \frac{1}{2}$ & 2 & $12 \frac{1}{2}$ & $\mathbf{N}$ & C.V. \& headaches & - & + & b & Nervous \\
\hline 83 & $\mathrm{~F}$ & $9 \frac{1}{2}$ & 4 & 1 & 4 & 2 & SN & $\begin{array}{l}\text { C.V. and pains in } \\
\text { legs and arms. }\end{array}$ & + & + & a & Normal \\
\hline 84 & $\mathrm{~F}$ & 8 & 4 & $1 \frac{3}{4}$ & 3 & 7 & SN & Rheumatics & 一 & + & c & Excitable \\
\hline 85 & $\mathbf{F}$ & 8 & 3 & $10 \frac{3}{4}$ & 2 & 13 & SN & $\begin{array}{l}\text { Abdomen pain and } \\
\text { headaches }\end{array}$ & + & + & c & Excitable \\
\hline 86 & M & 8 & 4 & $3 \frac{1}{2}$ & 4 & 0 & SN & $\begin{array}{l}\text { Cough and hoad- } \\
\text { aches. }\end{array}$ & - & + & b & Normal \\
\hline 87 & $\mathrm{~F}$ & 8 & 4 & $2 \frac{3}{4}$ & 4 & $2 \frac{1}{2}$ & $\mathrm{~N}$ & Headaches & - & + & b & Excitable \\
\hline 88 & F & 7 & 4 & $4 \frac{1}{2}$ & 4 & $8 \frac{1}{2}$ & $\mathrm{~N}$ & $\begin{array}{l}\text { C.V. and ? rheu- } \\
\text { matic pains }\end{array}$ & - & t & b & Normal \\
\hline 89 & M & $8 \frac{1}{2}$ & 3 & 11 & 3 & 4 & SN & C.V. & 一 & + & a & Normal \\
\hline 90 & M & 9 & 4 & $3 \frac{1}{2}$. & 3 & $13 \frac{1}{2}$ & SN & Constipation & + & + & c & Phlegmatic \\
\hline 91 & $\mathrm{~F}$ & 6 & & & 2 & $12 \frac{1}{4}$ & $\mathrm{~N}$ & Night terrors & + & + & c & Excitable \\
\hline 92 & $\mathrm{~F}$ & 8 & 4 & $1 \frac{3}{4}$ & 4 & 0 & $\mathrm{~N}$ & T.T. & - & - & c & Excitable \\
\hline 93 & $\mathbf{F}$ & 11 & 4 & 6 & 4 & $12 \frac{1}{4}$ & $\mathrm{~N}$ & Pains in back & - & + & c & Normal \\
\hline 94 & F & 8 & 3 & $11 \frac{1}{2}$ & 3 & $6 \frac{1}{2}$ & $\mathbf{N}$ & $\begin{array}{l}\text { Headaches and ? } \\
\text { rheumatic pains. }\end{array}$ & - & + & b & Excitable \\
\hline 95 & $\mathrm{M}$ & $7 \frac{1}{2}$ & & & 3 & $6 \frac{1}{4}$ & SN & $\begin{array}{l}\text { Wasting, 'back- } \\
\text { wardness.' }\end{array}$ & - & + & c & Normal \\
\hline 96 & $\mathbf{M}$ & $7 \frac{1}{2}$ & & & & & SN & Pains in knees & - & - & b & Normal \\
\hline 97 & $\mathbf{M}$ & $6 \frac{1}{2}$ & & & & & $\mathbf{N}$ & C.V. & - & + & b & Normal \\
\hline 98 & M & 9 & 4 & $1 \frac{3}{4}$ & 3 & 8 & $\mathrm{SN}$ & Cough & + & + & b & Normal \\
\hline 99 & $\mathbf{F}$ & 7 & 3 & $10 \frac{1}{2}$ & 3 & 0 & SN & Cough & + & + & c & Excitable \\
\hline 100 & $\mathrm{~F}$ & $6 \frac{1}{2}$ & 3 & $5 \frac{1}{2}$ & 2 & 6 & SN & Fidgets & + & + & c & Excitable \\
\hline 101 & M & 9 & 4 & $5 \frac{3}{4}$ & 4 & 2 & $\mathrm{~N}$ & Headaches & - & + & c & Normal \\
\hline 102 & $\mathbf{F}$ & 7 & 3 & 10 & 3 & 6 & $\mathbf{N}$ & Urticaria papulasa & + & + & b & Excitable \\
\hline
\end{tabular}




\begin{tabular}{|c|c|c|c|c|c|c|c|}
\hline Habit & Complexion & Remarks & $\mid \begin{array}{l}\mathrm{Plasma}_{3} \\
\mathrm{NaHCo}_{3}\end{array}$ & $\begin{array}{c}\text { Plasma } \\
\mathrm{NaCl}\end{array}$ & $\begin{array}{l}\text { Blood } \\
\text { sugar }\end{array}$ & $\begin{array}{c}\text { Rothera } \\
\text { urine }\end{array}$ & $\begin{array}{l}\text { Urinary } \\
\text { chloride }\end{array}$ \\
\hline Normal & Good & & .0293 & .588 & & - & \\
\hline Thin & Pale & $\begin{array}{l}\text { Had growing pains. Tonsillec- } \\
\text { tomy } 5 \text { years ago }\end{array}$ & .0295 & .590 & & - & \\
\hline Normal & Good & $\begin{array}{l}\text { ? subacute rheumatism ; slight } \\
\text { systolic bruit apex. }\end{array}$ & .0270 & .608 & & - & $1 \cdot 14$ \\
\hline Normal & Pale & Train sick formerly & .0303 & $\cdot 605$ & & - & $1 \cdot 16$ \\
\hline Normal & Good & & .0295 & 600 & & - & 32 \\
\hline Thin & Pale & Train sick & .0303 & .603 & & sl. + & $1 \cdot 12$ \\
\hline Thin & Pale & Has eyclical vomiting & .0292 & .629 & & sl. + & $1 \cdot 14$ \\
\hline Thin & Pale & $\begin{array}{l}\text { Tonsillectomy } 3 \frac{1}{2} \text { years pre- } \\
\text { viously. }\end{array}$ & .0291 & .588 & & ++ & $1 \cdot 08$ \\
\hline Normal & Good & & .0311 & .604 & & - & .506 \\
\hline Thin & Good & & .0292 & .620 & & - & .111 \\
\hline Normal & Good & $\begin{array}{l}\text { Extreme nervousness ? rheu- } \\
\text { matic pains. Heart normal. }\end{array}$ & .0263 & .611 & & - & $1 \cdot 18$ \\
\hline Normal & Pale & $\begin{array}{l}\text { Train sick. Tonsillectomy } 1 \frac{1}{2} \\
\text { years ago. Urticaria papu- } \\
\text { losa. }\end{array}$ & .0288 & .614 & & 一 & \\
\hline Thin & Good & $\begin{array}{l}\text { Heart ? slightly enlarged, no } \\
\text { murmurs. }\end{array}$ & .0270 & 600 & & +- & 687 \\
\hline Normal & Pale & Train sick & .0308 & $\cdot 603$ & & - & .06 \\
\hline Normal & Good & Wets bed & .0254 & $\cdot 614$ & & - & .995 \\
\hline Normal & Good & & .0288 & .618 & & 一 & .664 \\
\hline Normal & Good & Heart normal & .0294 & $\cdot 603$ & & - & $1 \cdot 14$ \\
\hline Very thin & Good & $\begin{array}{l}\text { Train sick. Tonsillectomy } 3 \frac{1}{2} \\
\text { years ago. }\end{array}$ & .0228 & .598 & & 一 & 1.21 \\
\hline Normal & Pale & & .0264 & .607 & & ++ & .658 \\
\hline Normal & Good & & .0294 & 606 & & - & $\cdot 589$ \\
\hline Normal & Good & & .0299 & .603 & & sl. + & .679 \\
\hline Normal & Good & Train sick & .0306 & $\cdot 608$ & & - & 1.05 \\
\hline Normal' & Good & $\begin{array}{l}\text { Heart normal. Had ? rheu- } \\
\text { matic fever } 2 \text { years ago. }\end{array}$ & $\cdot 0296$ & $\cdot 597$ & & - & $\cdot 326$ \\
\hline Normal & Good & & .0284 & $\cdot 611$ & & sl. + & $\cdot 401$ \\
\hline Normal & Good & & .0278 & .591 & & - & \\
\hline Normal & Good & & .0300 & 605 & & - & $\cdot 393$ \\
\hline Thin & Pale & $\begin{array}{l}\text { Train sick. Systolic bruit at } \\
\text { apex. }\end{array}$ & .0278 & .576 & & - & $\cdot 439$ \\
\hline Thin & Pale & C.V. & .0245 & .603 & & - & .907 \\
\hline Thin & Pale & & .0257 & .617 & & - & .890 \\
\hline Thin & Good & & .0315 & .611 & & - & .962 \\
\hline Normal & Good & & .0299 & .594 & & + & .965 \\
\hline
\end{tabular}


TABLE I-continued.

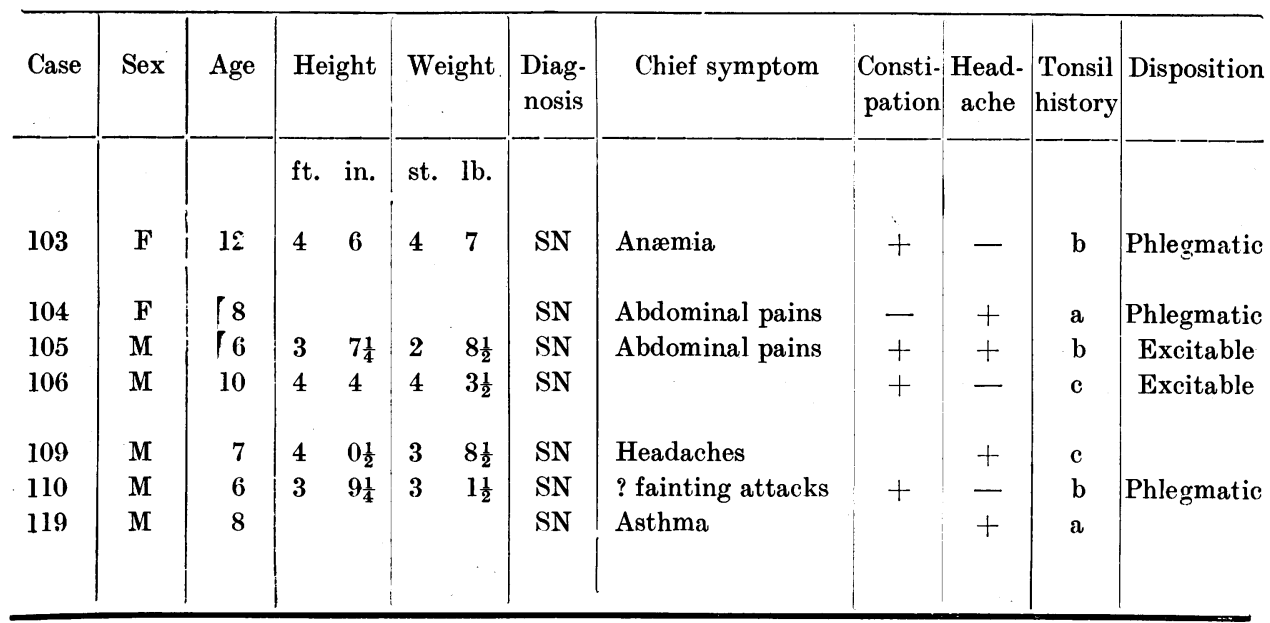

elsewhere, and not a deficiency of sugar in the blood. To summarize the findings in these two groups therefore, it may be said that in subnormal, or debilitated children, there is a definite acidosis, with a tendency to high plasma chlorides, and no evidence of hypoglycæmia.

TABLE 2.

COMPARISON OF NORMAL AND SUBNORMAL GROUPS.

\begin{tabular}{|c|c|c|c|c|c|c|c|c|c|}
\hline \multirow{2}{*}{ Type } & \multicolumn{3}{|c|}{ Blood sugar (mgrm. \%) } & \multicolumn{3}{|c|}{$\mathrm{NaHCO}_{3}$ (molar) } & \multicolumn{3}{|c|}{$\mathrm{NaCl}$ (grm. \%) } \\
\hline & No. of & Av. & Range & No. of & Av. & Range & No. of & Av. & Range \\
\hline Normal & 11 & 95 & $77-139$ & 38 & .0299 & $\cdot 0263-.0321$ & 24 & $\cdot 605$ & $\cdot 587-638$ \\
\hline Subnormal & 25 & $100 \cdot 6$ & $78-146$ & 70 & .0276 & $\cdot 0228-0316$ & 40 & $\cdot 610$ & $\cdot 576-659$ \\
\hline
\end{tabular}

Acidosis not due to ketosis.-Owing to the frequency with which acetonuria occurs in sick children, it has generally been assumed that the acidosis so commonly found in them, is due to excess of ketone bodies in the blood. In fact, the terms ketosis, acetonæmia, and acidosis, have long been used synonymously by clinicians. Frew ${ }^{7}$ has shown that something like $60 \%$ of children entering hospital for various conditions have acetonuria on admission, and this high incidence has been confirmed by others. But it must be remembered that all these children are sick, and many of them pyrexial. Acetonuria is almost constantly present too throughout the earlier stages of the acute exanthemata, such as scarlet fever, measles and diphtheria, and this is usually accompanied by some degree of acidosis (decreased plasma bicarbonate). As a rule, however, even in the acute exanthemata, the amount of acetone bodies in the blood is not sufficient to account for the 


\begin{tabular}{|c|c|c|c|c|c|c|c|}
\hline Habit & Complexion & Remarks & $\begin{array}{l}\text { Plasma } \\
\mathrm{NaHC}^{\circ} \mathrm{O}_{3}\end{array}$ & $\begin{array}{c}\text { Plasma } \\
\mathrm{NaCl}\end{array}$ & $\begin{array}{l}\text { Blood } \\
\text { sugar }\end{array}$ & $\begin{array}{c}\text { Rothera } \\
\text { urine }\end{array}$ & $\begin{array}{l}\text { Urinary } \\
\text { chloride }\end{array}$ \\
\hline Thin & Very pale & $\begin{array}{l}\text { Train sick. Tonsillectomy } 3 \\
\text { years previously. }\end{array}$ & .0278 & 588 & & + & 994 \\
\hline Thin & Very pale & Simulating appendicitis & .0263 & .614 & & sl. + & $1 \cdot 32$ \\
\hline Thin & Good & & .0280 & .638 & & - & \\
\hline Thin & Pale & $\begin{array}{l}\text { Rheumatic fever } 15 \text { months } \\
\text { ago. Mitral regurgitation. }\end{array}$ & .0276 & .612 & & - & 396 \\
\hline Flabby & Pale & & $\begin{array}{l}.0278 \\
.0274\end{array}$ & $\begin{array}{r}.588 \\
.601\end{array}$ & & - & $\begin{array}{r}1.26 \\
.58\end{array}$ \\
\hline Normal & Good & $\begin{array}{l}\text { Tonsillectomy } 3 \text { years pre- } \\
\text { viously. }\end{array}$ & .0272 & .600 & & - & \\
\hline
\end{tabular}

whole of the decrease in the plasma bicarbonate: in other words, the whole of the acidosis is not due to ketone bodies ${ }^{8}$. Our observations on subnormal children suggest that the acidosis which is so frequently found, is practically never due to an associated ketonæmia. The evidence for this statement is as follows:-

(a) Of the total 108 cases, a positive Rothera reaction was found in the urine at the time of the blood examination in only 17 cases, 1 normal, and 16 subnormals (i.e., 16.5 per cent.). A positive ferric chloride test was present in none of the cases.

(b) Table 3 shows the chemical findings in the cases in whom a positive Rothera reaction was found in the urine. Comparing the figures in Tables 2 and 3 it is seen that in the acetonuric children, the average plasma bicarbonate was even a little higher than the average for the group (i.e., subnormals), namely, $0.0280 \mathrm{M}$, compared with $0.0276 \mathrm{M}$. It will also be observed that the blood sugar in the acetonuric and non-acetonuric children is practically the same, namely, $99 \mathrm{mgrm}$. per cent. in the former, and $100 \cdot 6 \mathrm{mgrm}$. per cent. in the latter.

TABI.E 3.

Cases silowing Rothera reaction in tile vidne.

\begin{tabular}{|c|c|c|c|c|c|c|c|c|c|}
\hline \multirow{2}{*}{ Type } & \multicolumn{3}{|c|}{ Blood sugar (mgrm. \%) } & \multicolumn{3}{|c|}{$\mathrm{NaHC}^{\prime}()_{3}$ (molar) } & \multicolumn{3}{|c|}{$\mathrm{NaCl}(\mathrm{grm} . \%)$} \\
\hline & $\begin{array}{c}\text { No. of } \\
\text { cases }\end{array}$ & Av. & Range & $\begin{array}{c}\text { No. of } \\
\text { cases }\end{array}$ & Av. & Range & $\begin{array}{c}\text { No. of } \\
\text { cases }\end{array}$ & Av. & Range \\
\hline Normal & - & - & - & 1 & .0299 & - & 1 & .594 & 一 \\
\hline Subnormal & 3 & 99 & $90-114$ & 16 & .0285 & $\cdot 0248-0303$ & 9 & .608 & $\cdot 588$ — 6332 \\
\hline
\end{tabular}


It is clear then that in the debilitated child the presence of absence of acetonuria furnishes no guide to the degree of acidosis.

(c) In a study of a large number of cases of scarlet fever, diphtheria, measles, typhoid, and streptococcal tonsillitis ${ }^{8}$, in which repeated estimations of the plasma bicarbonate weie carried out at regular intcrvals throughout the course of the illness, it was found, as noted above, that estimations of the total acetone bodies in the blood, even in the early and pyrexial stages of the disease during which acetonuria was present, showed that the acidosis was not nearly accounted for by the quantity of these substances present in the blood. Further, it was shown that in 25 per cent. of cases of scarlet fever, a late or delayed acidosis occurred, often as marked in degrce as the primary or early acidosis; and that this late form of the condition, which generally occurred between the 15th and the 30th days of the disease, was not accompanied by acetonuria.

(d) In the apyrexial subrormal children with acetonuria in the present series, an attempt was made to estimate the total acetone bodies in the blood of four cases. In none of them was it possible to obtain any evidence of ketonæmia, in fact, no more precipitate was formed than in the 'blank' estimations. On these grounds therefore it is concluded that the acidosis of debility is not due to ketosis.

In this connection, it is of intcrest to note the difference in response to treatment with sugar, between the apyrexial subnormal child with or without acetonuria, and the child suffering from acute pyrexial disease, such as scarlet fever. In the former, with a normal blood sugar (sce above), the giving of extra sugar by mouth usually produces a rapid and striking improvement in tho general health ${ }^{6}$; whereas in the latter, where the blood sugar tends if anything to be above normal, not only does the giving of sugar usually fail to produce any improvement in the condition of the child, but it does not hasten the disappearance of acetone bodies from the urine. This suggests that during acut pyrexial disorders, there is inability to utilize the sugar : whereas in debility, although the blood sugar is normal (it is presumably of the first importance to maintain a normal concentration of sugar in the blood), the glycogen reserves of the body are on the verge of exhaustion.

Before leaving the subject of the biochemical variations in the two groups of children investigated, it may be of interest to cite a few of the individual cases, as illustrating some of the points already raised. It must be emphasized that although a decreased plasma bicarbonate is the rule in debilitated children, the degree of acidosis is not necessarily a measure of the unfitness in any given case, though there is a rough parallelism between the two factors. Case 82 looked in every way in the most perfect health, yet he had a plasma bicarbonate of only $0.0263 \mathrm{M}$. Case 18 , on the other hand, looked extremely unwell, had a strong positive Rothera in the urine, and a plasma bicarbonate of $0.0290 \mathrm{M}$. The relation between the blood sugar and the degree of fitness is of special interest, in view of the current teaching to which reference has already been made. The children who appeared to be mast unfit, and were pallid, exhausted, and lacking in energy and in interest in their surroundings, 
were not those with the lowest blood sugars, but, on the contrary, it was quite often found that their blood sugar was relatively high, even above normal. This was particularly noticeable in Cases 10 to $16,18,19,20,25$, and others. Further, repeated estimations of the blood sugar at fortnightly intervals on some of these cases, failed to show any more 'instability' in the bloodsugar level in the one group than in the other. No useful purpose would be served by multiplying instances of these discrepancies, they all go to show that isolated observations of this kind are of little value, and that individual differences are so marked, that it is only in the study and comparison of groups of similar cases, that much can be learned by our present rather crude methods.

\section{Relation of tonsillar disease to debility in children.}

Disease of the tonsils and of the upper respiratory tract in general, has so often been considered as one of the chief causes of debility in children, that the removal of tonsils and adenoids is now regarded as almost a necessary preliminary, if not the only treatment required in these cases. It was considered desirable, therefore, to assess as far as possible the relation of tonsillar disease to the clinical and biochemical findings in the present series of cases.

In 94 children, 35 normals and 59 subnormals, the state of the tonsils, and the history of tonsillar disease, including sore throats, cervical adenitis, and chronic otorrhœea, were carefully noted. Table 4 shows the state of the tonsils as found in the healthy and debilitated children. The notation used is that described previously.

TABLE 4.

Condition of tONSILS IN CASES EXAMINED.

\begin{tabular}{|c|c|c|c|c|c|}
\hline & & & A. & B. & C. \\
\hline & Normal .. & . & 5 & 16 & 14 \\
\hline & Subnormal .. & .. & 17 & 21 & 20 \\
\hline
\end{tabular}

A study of Table 4 shows that :-

(1) of 30 normal children, approximately 50 per cent. had tonsillar disease, and 50 per cent. did not.

(2) of 42 subnormal children, approximately 50 per cent. had tonsillar disease, and 50 per cent. did not.

(3) of 22 children with previous tonsillectomy, 17 or 77 per cent. were still subnormal, that is, suffering from varying degrees of debility, from one to eight years after tonsillectomy, despite no history of subsequent throat disorders.

The chemical findings in 70 of the children in whom the question of tonsillar disease was studied, are shown in Table $\tilde{5}$. 
TABLE 5.

\begin{tabular}{|c|c|c|c|c|c|c|c|c|c|}
\hline \multirow{2}{*}{ Type } & \multicolumn{3}{|c|}{ Blood sugar (mrgm. \%) } & \multicolumn{3}{|c|}{$\mathrm{NaHC}^{\prime} \mathrm{O}_{3}$ (molar) } & \multicolumn{3}{|c|}{$\mathrm{Na}\left({ }^{\prime} \mathrm{l}(\mathrm{grm} . \%)\right.$} \\
\hline & $\begin{array}{c}\text { No. of } \\
\text { cases }\end{array}$ & Av. & Range & $\begin{array}{l}\text { No. of } \\
\text { cases }\end{array}$ & Av. & Range & $\begin{array}{c}\text { No. of } \\
\text { cases }\end{array}$ & Av. & Range \\
\hline $\begin{array}{c}\text { (b) } \\
\text { Tonsils } \\
\text { healthy }\end{array}$ & 8 & 88 & $77-103$ & 36 & .0287 & $.0254-.0316$ & 23 & .607 & $.576-638$ \\
\hline $\begin{array}{c}(c) \\
\text { Tonsils } \\
\text { unhealthy }\end{array}$ & 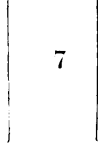 & $99 \cdot 7$ & $89-116$ & 34 & .0286 & $.0245-0315$ & 22 & $\cdot 619$ & $.588-.632$ \\
\hline
\end{tabular}

It will be seen that there is no significant difference between those with, and those without, tonsillar disease, as regards the chemical findings in the blood.

Table 6 shows the results of similar observations on the 2.2 children who had had a previous tonsillectomy. Here again, it will be seen that there is very little difference between subnormal children who have had a previous tonsillectomy, and subnormal children who have not, though it must be admitted that the plasma bicarbonate is a little higher in the former, than in the latter. It is interesting in this connection to recall the evidence regarding the results of tonsillectomy in such specific diseases as acute rheumatism and acute nephritis. It has been shown by many investigators, that this operation is of extremely doubtful value in both diseases, especially as regards recurrences, indicating that this operation does not generally lead to sufficient improvement in general health to protect against recurrence of the disease, once infection has occurred, although in both these diseases the tonsils themselves probably provide the primary portal of entry for the infection.

TABLE 6.

Toxsillectomizei cases.

\begin{tabular}{|c|c|c|c|c|c|c|c|c|c|}
\hline \multirow{2}{*}{ Type } & \multicolumn{3}{|c|}{ Blood sugar (mgrm. \%) } & \multicolumn{3}{|c|}{$\mathrm{NaH}\left(\mathrm{CO}_{3}\right.$ (molar) } & \multicolumn{3}{|c|}{$\mathrm{NaCl}(\mathrm{grm} . \%)$} \\
\hline & $\begin{array}{l}\text { No. of } \\
\text { cases }\end{array}$ & Av. & Range & $\begin{array}{l}\text { No. of } \\
\text { cases }\end{array}$ & Av. & Range & $\begin{array}{c}\text { No. of } \\
\text { cases }\end{array}$ & Av. & Range \\
\hline Normal & - & - & - & 4 & .0301 & $\begin{array}{l}.0312 \\
.0295\end{array}$ & 5 & $\cdot 596$ & $\begin{array}{l}.608 \\
.587\end{array}$ \\
\hline Subnormal & 3 & 101 & $\begin{array}{r}109 \\
92\end{array}$ & 18 & .0279 & $\begin{array}{l}.0303 \\
.0228\end{array}$ & 13 & .610 & $\begin{array}{l}.632 \\
\cdot 588\end{array}$ \\
\hline Total & 3 & 101 & $\begin{array}{r}109 \\
92\end{array}$ & 22 & .0290 & $\begin{array}{l}.0312 \\
.0228\end{array}$ & 17 & $\cdot 603$ & $\begin{array}{l}\cdot 632 \\
\cdot 587\end{array}$ \\
\hline
\end{tabular}


Paterson and Gray $^{9}$ showed that in a series of subnormal children, marked improvement in general health, and increase in weight, was found up to 6 months after tonsillectomy, a result which, at first sight, strongly suggests the importance of tonsillar disease as a cause of chronic debility in children. The Board of Education ${ }^{10}$, however, found that if a series of subnormal children in whom the tonsils had previously been removed, were followed up for a considerably longer period after operation than six months, the initial improvement noted by Paterson and Gray, was not maintained. Further evidence relating to this problem has been reported by one of us (H.G.C.) elsewhere ${ }^{11}$.

The balance of evidence would seem to suggest that tonsillar disease is not such an important factor in the production of chronic debility in children as is usually supposed, and that tonsillectomy is an operation which is often performed unnecessarily.

\section{Relation of debility to cyclical vomiting.}

In a paper already referred to above ${ }^{6}$, it was pointed out that cyclical vomiting was a disorder met as frequently amongst the children of the hospital classes as amongst those of the well-to-do. This, again, is contrary to the usual teaching. It was also stated that cyclical vomiting occurred in about 30 per cent. of all elementary school children. The evidence for this was based on a history of the symptom-complex described above in 500 consecutive children between the ages of 6 to 12 years, attending hospital for minor disorders. In actual fact, cyclical vomiting, as judged by these standards, occurred in 45 per cent. of the series referred to, but in order to eliminate possibility of error arising from the necessity of asking leading questions in a number of instances, an allowance of 15 per cent. was made. The figure of 30 per cent., therefore, may be taken as a conservative estimate of the frequency of this disorder in the children of the hospital classes. E. C. Warner, working in the Children's Department at Guy's Hospital, informs us in a personal communication that, adopting the same criteria as we had done, he had found that 20 per cent. of a series of 300 children out-patients, between the ages of 3 to 12 years inclusive, suffered from major attacks of cyclical vomiting. As this disorder does not usually appear in its most typical form before six years of age, it is clear that, allowing for this, 30 per cent. is again a fair estimate of the frequency of cyclical vomiting in children of this social class.

In the present series of 108 children, a definite history of major attacks of cylical vomiting was obtained in 47 cases. Table 7 compares the biochemical findings in 16 children with a history of cyclical vomiting, who had not recently had an attack, and 14 subnormal children who gave no history of this disolder.

These results show that there is no biochemical difference, in respect of the observations made, between these two groups. Of the 39 normal children, 13 to 33 per cent., gave a history of cyclical vomiting, and of the 70 subnormal children, 35 or 50 per cent., suffered from this disorder. These figures, though not very large, suggest that cyclical vomiting is more often found in the unfit, 
than in the fit child, and still further emphasizes the clinical fact, hitherto overlooked, of the frequency with which this condition is met in children of the poorer classes.

TABLE 7.

Comparison of CASES ShOWING CYClical vomiting WITH SUBNORMal CASES Without it.

\begin{tabular}{|c|c|c|c|c|c|c|c|c|c|}
\hline \multirow{2}{*}{ Type } & \multicolumn{3}{|c|}{ Blood sugar (mgrm. \%) } & \multicolumn{3}{|c|}{$\mathrm{NaHCO}_{3}$ (molar) } & \multicolumn{3}{|c|}{$\mathrm{NaCl}$ (grm. \%) } \\
\hline & $\begin{array}{l}\text { No. of } \\
\text { cases }\end{array}$ & Av. & Range & $\begin{array}{c}\text { No. of } \\
\text { cases }\end{array}$ & Av. & Range & $\begin{array}{c}\text { No. of } \\
\text { cases }\end{array}$ & Av. & Range \\
\hline C.V. & 16 & $99 \cdot 7$ & $75-146$ & 30 & .0277 & $.0228-0316$ & 10 & $\cdot 610$ & $\cdot 576-638$ \\
\hline G. & 14 & $98 \cdot 6$ & $77-139$ & 35 & .0279 & $.0254-0316$ & 17 & .601 & $\cdot 588$ - 623 \\
\hline
\end{tabular}

It is a matter of common observation, referred to by all authorities on the subject, that cyclical vomiting is most apt to occur and to be most intractable, in the highly-strung, excitable child, and to this extent the biochemical results given in Table 7, may be regarded as typical of the nervous child; but as mentioned elsewhere ${ }^{6}$, cyclical vomiting is a disorder which occurs perhaps in its most dramatic form in the child of nervous make-up, but it is by no means entirely confined to such. This is opposed to the view expressed by some that the acidosis and disturbances of carbohydrate and fat metabolism, with which it is said to be associated, constitute a biochemical picture peculiar to the nervous child.

It is at least apparent that cyclical vomiting is a disorder of very frequent occurrence amongst children of all social classes; that children who suffer from it do not differ in respect of the biochemical observations made herein, from those who do not, and that it is not the prerogative of the nervous child.

\section{Observations on the treatment of debility in children.}

About seven years ago, one of us (A.A.G.), when collecting cases of cyclical vomiting for investigation in the Children's Department at Guy's Hospital, observed that those children who had been given extract of malt, largely with the object of securing regular attendance at out-patients, ceased to have further attacks of the disorder. This at once suggested that sugar in some form was the needed antidote. This hypothesis was rendered more probable, by the fact that, in an undue proportion of these cases, it was noticed that sugar was being withheld, as far as possible, as the mother had been told that it was ' bad for the teeth,' ' brought the child out in spots,'. or, ' was bad for children.' a doctrine which incidentally, is still held, and acted upon, even in some of the best public schools in the country. There is, of course, no adequate scientific evidence for any of these suggestions.

In order to test further the action of sugar in preventing cyclical vomiting, a group of 30 children suffering from this disorder was given extra. sugar; 
and kept under observation for from one to three years, and as a result, it was found that with very few exceptions, attacks of cyclical vomiting ceased to occur. It became apparent too, that not only was cyclical vomiting easily prevented by this simple and harmless measure, but that a large number of the other common symptoms of debility in children, such as headaches, recurrent abdominal pains, constipation, ' growing pains,' pallor, and undue fatiguability, were also cured, and prevented, and that the giving of extra sugar, in any form, quite frequently resulted in a rapid and marked improvement in the general health, spirits, and appearance of the child. In fact, without any other form of treatment, or alteration in the daily régime, a pale, dispirited, ailing child, would often be converted into a high spirited, healthy, and happy being. This form of treatment was later adopted by Dr. H. C. Cameron ${ }^{13}$, ${ }^{14}$, and he has confirmed the beneficial results obtained. As pointed out elsewhere ${ }^{6}$, cyclical vomiting occurs in three main forms:-the classical cyclical, or periodic vomiting, cyclical diarrhœea, and cyclical pyrexia, each of which may occur singly or together. Quite early in the investigation, it was noticed that there was also a recurrent, or cyclical, ' respiratory' group, the symptoms of which were indistinguishable from those of ordinary bronchial asthma. In view, however, of the well known difficulty of differentiating between some cases of asthma and recurrent bronchitis in young children, it was considered unwise to assert that asthma could be successfully treated by sugar, but it soon became obvious that these cases were, in fact, true examples of bronchial asthma, and that at least in some of them, sugar was an effective prophylactic. Although it has been shown that the most common and constant biochemical feature of debility in children is an 'acidosis,' it is a remarkable fact that, in our experience, the giving of alkalies by mouth does not in any way lead to improvement in the condition, or in any of its numerous and varied symptoms. As far as we have been able to ascertain, also, alkalies are quite without value in the treatment or prevention of cyclical vomiting and asthma in children. There is, however, one point in this connection, which should be borne in mind, before concluding that alkalies are without value in these cases, and that is, the question of increased liability to infection, where some degree of acidosis is present. Cameron ${ }^{12}$ asserts that children suffering from acidosis are predisposed thereby to infection. We have long had this possibility in mind, and have endeavoured to obtain evidence upon the question, but at present, it can only be said that no conclusions have been reached. If the type of acidosis referred to here should be shown to predispose to infection, then it is possible that the use of alkalies in these cases may be justified.

\section{Conclusions.}

(1) The state of subnormal health in children, usually called 'debility,' is generally associated with some degree of ' acidosis,' defining this as a reduction of the plasma bicarbonate only.

(2) Debility in children is not commonly accompanied by hypoglycæmia, and the term 'glycopenia' is inaccurate, and should not be used to describe these cases. 
(3) The acidosis of debility is not generally due to an associated acetonæmia or ketosis.

(4) Ketosis in children is not generally associated with hypoglycæmia.

(5) Tonsillar disease is not the most common cause of debility in children, and tonsillectomy is an operation which is often performed unnecessarily.

(6) Cyclical vomiting is a disorder which occurs with great frequency amongst children of the so-called hospital classes, the incidence in this class being about 30 per cent. of all children between the ages of 6 to 12 years inclusive.

(7) The biochemical picture associated with disturbances of the carbohydrate and fat metabolism, acetonæmia, etc., is not more frequently found in 'nervous,' than in other subnormal children. child.

(8) There is no specific biochemical picture peculiar to the 'nervous'

(9) There is no evidence that alkalies are of value in the treatment of debility in children.

We are much indebted to Prof. R. Donaldson and Dr. Arthur Davies, Directors of the Pathological Departments of Guy's Hospital, and Queen Mary's Hospital, respectively, for laboratory facilities, without which we should not have been able to undertake this investigation. Also to the Medical Research Council for a grant with the aid of which this work was carried out.

\section{REFERENCES.}

1. Osman, A. A., \& ('lose, H. G. (in the Press).

2. Van Slyke, D. D., Stillman, E., \& Cullen, G. E., J. Biol. Chem., N.Y., 1919, XX XVII,167.

3. Claudius, Acta Med. Scand., Stockholm, 1924, LXI, 3.

4. Van Slyke, D. D., \& Fitz., F. W., J. Biol. Chem., N.Y., 1917, ХХХII.

5. Baird, M. M., Douglas, C. G., Haldane, J. B. S., \& Priestley, J. (i., Proc. Physiol. Soc., 1922-23, LVII, p. XLI, 1922-23.

6. Osman, A. A., Loc. cit., 1929, I, 150.

7. Frew, R. S., Lancet, Lond., 1911, ii, 1264.

8. Carter, H., Close, H. G., \& Osman, A. A. (in the Press).

9. Paterson, 1., \& Gray, G. W., Lancet, Lond., 1928, ii, 1074.

10. Board of Education. Interim Report of the Medical ('ommittee on Adenoids and Enlarged Tonsils, 1929.

11. C'lose, H. G., Guy's Hosp. Rep., Lond., 1930, LXXX, 45.

12. Cameron, H. C., Brit. Med. J., Lond., 1929, i, 185. 